\title{
A reflective approach for pediatric dental care at the university clinical school, in the time of COVID-19
}

\author{
Luciana de Barros Correia Fontes*, Marília Cleide Tenório Gomes, Amanda Caroline Henrique Mendes; Rosa \\ Maria Mariz de Melo Sales Marmhoud Coury, Leonardo Cavalcanti Bezerra dos Santos, Kátia Maria Gonçalves \\ Marques, Niedje Siqueira de Lima, Maria da Conceição de Barros Correia
}

Department of Clinical and Preventive Dentistry, Federal University of Pernambuco, Brazil

*Corresponding author: Luciana de Barros Correia Fontes, Department of Clinical and Preventive Dentistry, Federal University of Pernambuco, Brazil

\section{Opinion}

We are experiencing a unique time in everyone's life, globally, with an indefinite length of stay, as well as the extent of its impacts; the Coronavirus Disease (COVID-19) pneumonia pandemic. This disease is caused by the Severe Acute Respiratory Syndrome Coronavirus (SARS-CoV-2). As health workers, there are mixed feelings. On the one hand, hope, the frantic and urgent search for scientific evidences, prevention and efficient strategies for treatment of so many infected individuals with different levels of symptom severity. In another sense, the fear of contracting and acting as a vector for the transmission and dissemination of this disease. When analyzing the COVID-19 Worldwide Dashboard, the contamination and the number of deaths among health professionals is alarming; mainly if they work on the "front line" or with a risk of exposure to a high viral load, during management of the exposed person. And dentistry is no exception. It is necessary to prepare adjustments of dental practices; especially with undergraduate degrees treat children under the close supervision on faculty members on the Pediatric Dental Team. The participants in dental practice have a higher potential risk of infection, due to the face-toface communication and the exposure to saliva, blood, and other body fluids, because the procedures that are believed to generate aerosols and droplets and the handling of sharp instruments. There was a suspension of dental care services or a restriction on urgent or emergency cases, which comprise circumstances associated with pain, trauma or infectious processes; these bringing discomfort, impaired performance of oral functions, interventions necessary for critical medical procedures or that increase the risk of death to the patient. Gradually there is a greater openness to elective procedures.
At first, the sensation probably experienced by dentists would be related to not being considered as an essential professional when it comes to fighting together to "save lives". Is the oral health not associated with general health? If the oral cavity constitutes the "gateway" for many microorganisms and there are some oral manifestations of infections and diseases with systemic involvement in individuals; why were dentists not considered as essential workers? Here we do not question the professional skills of each specialty in the health area, but changes should be viewed as developments in multiprofessional health groups working together. Each university is preparing plans for reopening consistent and safe: restart and recovery research, education, and clinical services missions. In this context, the return to dental practice activities still represents a great challenge; a "new reality". And this perspective expands, particularly when we consider the dental care for children and adolescents. Here we are focused on children, because most of the time their possibility of understanding and clarification is still in development. Children need to interact to people and they should be "freedom" to play, explore and interact with environmental. Teaching experience, singularly in clinic-school for children in the University of Pernambuco College of Dentistry, in the Northeast of Brazil, needs a dynamics challenge. But how not to lose its essence? Playful and humanistic principles and procedures based on scientific evidence and technical improvement. We adopted some reinforces as positive facial expression, handshake, smile, hug and others. Everything supported by bioethics and human rights, in order to develop a better perception about dentists and dental care; to reduce tensions. 
Understanding of children and families, and their relationship to providing optimal pediatric dental care. We should construct strong interactions that allow healthy habits, cooperative behaviors, the reduction of fears and anxieties, good oral health conditions, adequate performance of orofacial functions and growth and craniofacial development with characteristics within a "normality" spectrum. Nowadays we are all (dental students, pediatric dental patients /family and staff) living in a world "in transformation", where insecurity, demands, competitions and hopelessness, associated with isolation or distancing from the other, can lead to high psychological distress. We need to implement measures to ensure the development of dental education and dental treatment, preserving the integrity of all those involved and to build the resilience during the COVID-19 pandemic. The demands for care have not stopped and there is a growing sense of anguish due to lack of dental care.
To Submit Your Article Click Here:

DOI: $10.32474 /$ IPDOAJ.2020.04.000183

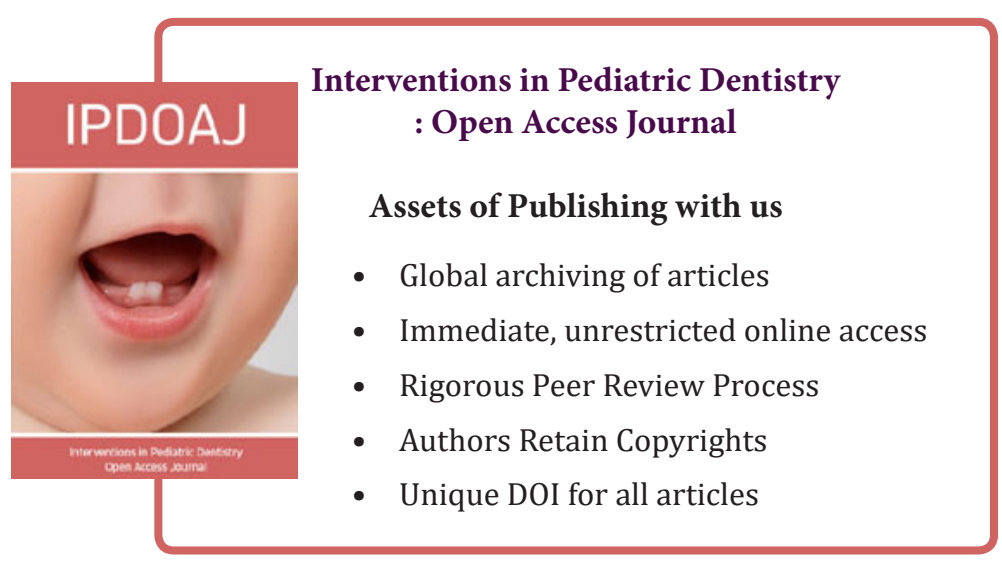

